\title{
Are patients' and doctors' accounts of the first specialist consultation for chronic back pain in agreement?
}

\author{
Kathy B White' \\ John Lee ${ }^{2}$ \\ Amanda C de CWilliams ${ }^{3}$ \\ 'Central and North West London \\ NHS Foundation Trust, ${ }^{2}$ School \\ of Life and Medical Sciences, \\ University College London, ${ }^{3}$ Research \\ Department of Clinical, Educational \\ and Health Psychology, Faculty of \\ Brain Sciences, University College \\ London, London, UK
}

This article was published in the following Dove Press journal: Journal of Pain Research

28 November 2016

Number of times this article has been viewed

Introduction: The first consultation at a specialist pain clinic is potentially a pivotal event in a patient's pain history, affecting treatment adherence and engagement with longer term selfmanagement. What doctors communicate to patients about their chronic pain and how patients interpret doctors' messages and explanations in pain consultations are under-investigated, particularly in specialist care. Yet, patients value personalized information about their pain problem. Patients and methods: Sixteen patients in their first specialist pain clinic consultation and the doctors they consulted were interviewed shortly after the consultation. Framework analysis, using patient themes, was used to identify full match, partial match, or mismatch of patient-doctor dyads' understandings of the consultation messages.

Results: Patients and doctors agreed, mainly implicitly, that medical treatment aiming at pain relief was primary and little time was devoted to discussion of self-management. Clinically relevant areas of mismatch included the explanation of pain, the likelihood of medical treatments providing relief, the long-term treatment plan, and the extent to which patients were expected to be active in achieving treatment goals.

Discussion: Overall, there appears to be reasonable concordance between doctors and patients, and patients were generally satisfied with their first consultation with a specialist. Two topics showed substantial mismatch, the estimated likely outcome of the next planned intervention and, assuming (as doctors but not patients did) that this was unsuccessful, the long-term treatment plan. It appeared that more complex issues often generate divergence of understanding or agreement. Despite the widespread recommendations to medical practitioners to check patients' understanding directly, it does not appear to be routine practice.

Conclusion: It is hoped that this research encourages more detailed examination of shared and divergent experiences of pain consultations and also their influence on the subsequent course of intervention and adherence to treatment (not addressed here).

Keywords: doctor-patient communication, pain clinic, patient information, biopsychosocial, rehabilitation

\section{Introduction}

Pain is considered to be chronic if it persists for longer than 3 months or beyond the point at which healing would be expected to be complete. ${ }^{1}$ Therefore, the diagnosis of chronic pain depends primarily on the patient's report. Chronic pain has a major negative impact on the quality of $\operatorname{life}^{2,3}$ and makes considerable demands on health services while gains from treatment may be relatively small. ${ }^{4}$ The back is the most common site of pain, affecting 1.6 million adults per year in the UK and costing an estimated $£ 18.7$ billion per year, mainly in work days lost. ${ }^{5}$

\footnotetext{
Correspondence: Amanda C de

C Williams

Research Department of Clinical, Educational and Health Psychology, University College London, Gower

Street, London WCIE 6BT, UK

Tel +44 2076791608

Fax +442079161989

Email amanda.williams@ucl.ac.uk
} 
The lack of identifiable damage or pathology and the repeated investigations and unsuccessful treatment attempts lead eventually to the diagnosis of chronic pain. For the patient, this diagnosis may conflict with the everyday model of pain where the cause is often evident and its resolution occurs with healing, with or without treatment, ${ }^{6-8}$ leading to disagreement between the patient and doctor on the nature of the pain problem. Patients' cognitive representations of their pain problem and the implications of those representations for appropriate treatment ${ }^{9}$ arise from coherent if mistaken ways of understanding the body and potential self-regulation in relation to pain. ${ }^{10}$ Models of psychosomatic or "medically unexplained" pain may be invoked, even though unsupported by evidence, ${ }^{11}$ further alienating patients who may assert even more strongly the need to find the elusive diagnosis. ${ }^{12,13}$ To establish a shared model, the clinician must engage with those representations and shift them toward a more veridical model of chronic pain and rehabilitation; effective communication in this process may reduce patient anxiety and increase the likelihood of patient adherence to advice and treatment recommendations, but it remains rare for clinicians to check patient understanding having provided their explanation of the problem. ${ }^{14}$ Chronic pain is adequately explained by changes in the central nervous system that are in part a function of the pain itself ${ }^{15}$ but may be prolonged by cognitive, emotional, and behavioral factors that are the target of rehabilitative treatment often within a pain service. ${ }^{16,17}$ The cognitive and behavioral perspective on pain aims to enable the person with pain to understand that chronic pain is not, like acute pain, a warning of imminent or actual damage but a dysfunction that need not prevent a return to a more satisfying lifestyle. ${ }^{18-20}$ Without agreement on the nature of the pain problem, the proposal that the patient should try to return to normal activities is often experienced as denial of the extent of their pain and disability, ${ }^{5,19}$

Therefore, the first consultation at a pain clinic may come after several years of unsuccessful attempts at diagnosis and treatment of presumed cause and at symptom relief. ${ }^{21,22}$ The specialist pain clinician aims to rule out treatable causes of pain, to identify any specialist analgesic methods that the patient may wish to try, and to assess the impact of pain on the patient's psychological and social well-being, which may be accessible to rehabilitative methods. This broad remit is ideally facilitated by an integrated team including psychology and physical therapy. ${ }^{5,23,24}$ The educative role of the pain clinician is increasingly recognized as important ${ }^{25-27}$ and valued by patients $;{ }^{28}$ it aims to enable the patient to change the patient's path from seeking a "cure" to learning how best to manage a chronic health problem. There is evidence that explanation to patients of their problems and possible treatments are a predominant reason for general practitioner and specialist consultation by patients with pain, ${ }^{14,29,30}$ and these improve patient satisfaction and adherence to treatment. ${ }^{31}$ However, trials of education alone are disappointing, ${ }^{32-35}$ and it remains unclear what content or process enables the chronic pain patient to change his or her understanding of pain and treatment options.

Therefore, we studied the first consultation for people with chronic back pain at a specialist pain clinic to which generalist or specialist doctors had referred them, aiming to identify the extent and content of agreement and disagreement between patient and doctor on the main messages of the medical consultation, including the treatment plan.

\section{Patients and methods \\ Setting and participants}

The research was conducted over 4 months in an adult specialist outpatient Pain Management Centre (PMC) in a major London teaching hospital (University College London Hospital). The research proposal was approved by The National Hospital for Neurology and Neurosurgery and Institute of Neurology Joint Research Ethics Committee representing the National Research Ethics Service (REC 08/H0716/73).

The multidisciplinary team offers assessments and interventions for patients with persistent pain, referred from primary and secondary health care. Referred patients are allocated according to next available appointment to one of the four specialty-trained pain doctors. The first medical consultation lasts 40-60 minutes with the aims of arriving at a provisional or definite diagnosis, explaining chronic pain, exploring therapeutic options, and agreeing upon a plan with the patient. Most patients are offered further appointments at the PMC for interventions or other therapies; the remaining patients are referred back to primary care with recommendations.

All patients who were being referred for the first time to the PMC for back pain were sent an information sheet inviting them to take part in the study, making it clear that they were under no obligation to do so. The researcher met with patients immediately before their appointments to discuss the study and consent.

In qualitative methodology, the researcher is considered part of the field of study ${ }^{36}$ The researcher (KW) who conducted and transcribed interviews was a White female in her late twenties, studying for a clinical psychology doctorate, not previously experienced in pain but using supervision and a reflective journal to critically evaluate her assumptions and practice. Her supervisor (ACW), who audited the analytic process, was an academic and clinical psychologist with long experience in pain. One of the participating doctors is also an author (JL) but was not involved in data analysis. 


\section{Patient interviews}

The researcher interviewed patients immediately after their specialist pain consultation using a semi-structured interview schedule that lasted from 25 to 50 minutes. Patients were encouraged to elaborate on their views, and the interview was audio-recorded and transcribed. The interview schedule consisted of open-ended questions including what patients thought the main messages of the consultation were; how the doctor explained their pain; what recommendations or advice the doctor gave about pain and treatment; and their own thoughts and feelings about the messages in the consultation (the interview schedule is available in the Supplementary material).

\section{Doctor interview}

All four doctors who assessed low back pain patients were invited to participate, and written informed consent was obtained from all participants. Each doctor was interviewed about each consultation with a study patient as soon as was practically possible, always within 3 hours of the consultation. Doctors' interviews lasted from 6 to 14 minutes. The semi-structured interview consisted of similar questions to those in the patient interviews: the main message(s) of the consultation; how pain was explained; and treatment recommendations, advice, and plan. In addition, doctors were asked how they thought patients understood and experienced the consultation messages and recommendations (the interview schedule is available in the Supplementary material).

\section{Data analysis}

Framework analysis (FA): patients' and doctors' understandings of the consultation messages

To identify the extent to which patients' and doctors' understandings of their consultation corresponded or diverged, patient and doctor transcripts were analyzed as dyads. In order to allow generalization across dyads, patient data were grouped in themes as given in the Results section. Since doctors' responses were relatively brief and concrete and frequently used the terminology in the interview questions, inductive approaches to analysis were inappropriate.

Analysis within dyads was performed using FA, a structured qualitative method..$^{37}$ Importantly, FA allowed comparison of doctors' and patients' accounts. FA guidelines ${ }^{38}$ were followed: identifying relevant data and generating a list of potential themes; iteratively developing a thematic framework to organize data; "indexing" all data using FA-derived themes; and rearranging indexed data for comparison within dyads (details available on request). Matches were defined as agreement at the level of subtheme(s) in patient material; partial matches and mismatches were defined by extent of disagreement and that disagreement identified. A subsample of dyad material was independently categorized by a second author and showed good concordance. Matches, partial matches, and mismatches were then tabulated unconstrained by the particular questions asked.

\section{Patient themes}

Patients' experience of the messages within the consultation was analyzed by thematic analysis (TA) of interview transcripts as part of a related study. ${ }^{39} \mathrm{TA}$ is an inductive, datadriven approach with minimal constraints on content. ${ }^{37}$ The researcher followed the thematic analysis guidelines, ${ }^{38,40-42}$ and the researcher and supervisor developed the analytic codes through an iterative and reflexive process.

\section{Results}

\section{Participants}

Of 24 patients invited to participate in the study, one declined, four did not attend their appointments, one had insufficient English for the interview, and two overruled their referral in asserting that back pain was not their primary problem. The remaining 16 , nine women and seven men, had a median age of 55 years (range 26-88 years). Ten were White British, with one each Black African, Black European-Caribbean, Asian British, Anglo-Indian, Indian, and Malaysian-Chinese. Nine were employed full- or part-time and seven were retired, one of whom identified himself as a carer. Median duration of chronic back pain was 6.5 years, with a range from 2 to 50 years.

The four doctors who participated had specialized in pain medicine for a mean of 13 years, with a mean of 7 years as a consultant. All were part of the same team, trained in the same model of consultations and involved in the same weekly interdisciplinary case discussions. The researcher's restricted availability on particular days created an uneven distribution such that seven patients were seen by doctor 1 , five by doctor 3 , and two each by doctors 2 and 4 . The two male and two female doctors' ages ranged from 37 to 60 years and identified themselves as White British, Sino-British, Indian, and Persian.

\section{Thematic analysis}

TA of patients' data is subsumed in the findings from the FA: it produced four themes that summarize the patients' understanding of their consultation with the doctors.

\section{Theme I: medical treatments are the answer}

The majority of patients (11/16) understood that their doctors recommended medical treatment, typically oral medication and/or analgesic injections, for their pain to be alleviated. These 
patients believed that the only interventions available were medical, and the doctor was pivotal in their treatment pathway.

So [the doctor] is going to try the nerve blocker, and then if the nerve blocker doesn't work, [the doctor] is going to try something different, and just keep going [...] if it doesn't work, just get more doctors' appointments. [P18]

\section{Theme 2: hope of pain relief against the odds}

All patients offered medical treatments restated the doctor's warning that treatment might not work, typically repeating the phrase "no guarantees". Despite this, most seemed hopeful that, even against the odds, the treatment would relieve their pain this time, while at the same time recognizing that they had little realistic basis for this, referring to "magic", "faith", and "a miracle".

[I'm] hoping for a magic wand and for them to do something to take the pain away. [P20]

It's a chance for me [...] if I do the injections and it works for me, it's like starting a new life again. [P1]

\section{Theme 3: the importance of trying all medical interventions first}

Most patients believed they needed to exhaust all medical interventions, whatever their own doubts, and some emphasized the importance of knowing that they had tried all that the doctor had recommended.

I will try anything; whatever [the doctor] asks me to take, I will take it. [P4]

I don't want to walk out of here, or anywhere, knowing that I didn't try. [P10]

\section{Theme 4: preference for self-management as treatment plan}

Patients' descriptions of doctors' treatment recommendations fell into two main categories: the only or main treatment recommendation was medical and the only or main treatment recommendation was rehabilitative pain management using exercise, pacing, and adapting activities; psychological intervention, although an important part of the rehabilitation package, was referred to very briefly, if at all. The five patients in the group recommended rehabilitation were all already using some pain management strategies and were relieved to receive recommendations consistent with their own choice.

[The doctor] pretty much verified more or less what I feel I am capable of $[. .$.$] this is what I feel and that is the expert,$ and you put the two together and you got to be doing the right thing. I am happy with that. [P8]

I am now thinking about lifestyle changes, even if it means making some uncomfortable decisions about how I live my life $[\ldots]$ at least then I can sustain it. [P14]

\section{Comparison between doctor and patient accounts: FA}

The FA examined how well the consultation's key messages were conveyed by examining the correspondence within patient-doctor dyads; Table 1 presents the full reports of matched and mismatched dyads across themes and subthemes.

\section{Summary of concordance between doctor-patient dyads}

This section addresses patients' understanding of the consultation messages, their expectations about long-term treatment plans, and their perceptions of the consultation. The degree of concordance was categorized as being matched, partially matched, or mismatched. A match was defined as the doctor's and the patient's account corresponding for all the main aspects of the subtheme. If a dyad was coded as partially matching or mismatching, the reason for the discrepancy was reported. There was total agreement on the next treatment and considerable agreement on its expected outcome.

Fifteen of 16 patients described feeling satisfied with all or most of their consultation, and there was much correspondence within doctor-patient dyads. However, since mismatch is of particular clinical interest, this section focuses on the four main areas.

\section{Mismatch of pain explanations}

Six of 13 dyads that discussed pain explanations differed on the doctor's explanation of pain. While patients in these dyads initially appeared confident in recounting the doctor's explanation of their pain, additional questioning revealed inconsistencies and lack of clarity. In four of these dyads, patients' explanations included the main aspects reported by the doctor, but with inconsistent additional details. For example, one doctor reported conveying to a patient that the pain was completely of muscular origin; the patient reported that the pain was due to a nerve and to weak muscles. Further, two doctors reported explaining to their patients that the pain was related to nerve function not to structural problems, but these patients reported explanations in terms of nerve and structural problems. Two further patients provided explanations that omitted a key element: 
Table I Matched and mismatched content in consultations

Theme, subtheme, and number of matches
I Doctors' explicit consultation messages
I.I Next agreed intervention: $16 / 16$ matches
I.2 Expectations of agreed intervention: $13 / 16$ matches

I.3 Doctor's explanation of pain: 7//3 matches

I.4 Patient's role in treatment: $2 / 7$ matches

2 Expectations about patients' long-term treatment

2.I Estimated likely outcome of the agreed intervention: 6/16 matches

2.2 Most likely long-term treatment plan: $9 / 16$ matches

3 Doctors' perceptions of patients compared with patients' own account 3.I Doctors' estimation of patients' understanding of consultation messages: $12 / 16$ matches

3.2 Doctors' impressions of patients' satisfaction with consultation: I I/ 6 matches

3.3 Doctors' descriptions of patients' pain and its impact: 0/6 matches

3.4 Doctors' descriptions of patients' agenda for the consultation: $0 / 7$ matches

3.5 Doctors' perceptions of patient's intention to adhere to advice: $4 / 6$ matches
Number and details of partial matches and mismatches

0 partial match or mismatch

3 partial matches: patients expected the treatment would be effective alone but the doctor stated that the treatment would only be effective when combined with physical therapy (2); patient did not report the doctor's statement that part of their pain was unlikely ever to improve (I). 0 mismatch

6 partial matches: patients reported additional elements of explanation inconsistent with the doctors' reports (4); patients omitted aspects of the explanation that the doctor had described as key (2).

0 mismatch

5 partial matches: patients described the general concept of pain management, but without details or inaccurately.

0 mismatch

10 mismatches: 8 patients reported significantly higher expectations of effectiveness than the doctor and 2 patients reported lower expectations. 7 mismatches: doctors reported that a pain management and/or physical therapy approach was most effective, while patients expected continued medical treatments.

4 partial matches: doctors overestimated the extent of agreement between their messages and patients' descriptions of messages received. 0 mismatch

2 partial matches: doctors underestimated patients' satisfaction.

3 mismatches: doctors overestimated patients' satisfaction.

6 mismatches: doctors described pain as mild that patients had described having significant impact on their daily lives or described patients as coping well who recounted struggling to cope.

7 mismatches: doctors described patients as having "fixed" ideas about desired treatment, while patients described changing some ideas about treatment as a result of the consultation (3); doctors reported that the patient only wanted injections, whereas patients stated that they wanted whichever treatment was most likely to improve their function (2); doctors believed explanation was unimportant to patients, whereas patients reported wanting clearer understanding of their pain (2).

I partial match: doctor underestimated the likelihood that the patient intended to adhere.

I mismatch: doctor overestimated the likelihood that the patient intended to adhere. for instance, one patient described "wear and tear", omitting the doctor's report describing the pain as a combination of the condition of the back and joints and of pressure on a nerve.

In the three consultations with no explanation of pain, two patients already had a consistent understanding of their pain from previous consultations, but the third patient provided an explanation for pain in structural terms that were inconsistent with the doctor's description of relevant pain mechanisms (P6).

\section{Mismatch of long-term treatment plans}

Nine of the 16 dyads were consistent on long-term treatment plans, whether for medical intervention that a doctor would provide or for nonmedical pain management strategies. The remaining seven dyads were mismatched on the most likely long-term treatment plan (subtheme 2.2: Table 1). All patients in these dyads were prescribed a medical intervention and reported expecting that other such medical interventions would follow if the next were unsuccessful. However, their doctors intended a medical intervention initially, providing some pain relief, followed by a long-term program of nonmedical pain management. Asked why they did not elaborate this plan to patients in the consultation, doctors reported postponing the discussion to the point where medical treatment was not effective, for two reasons: the doctor's wish to try to relieve pain, even if that was not the patient's preference, and acceding to the patient's preference despite their skepticism about benefit. 
I think first you need the physical because I believe people want that, [...] even if you have your suspicion it won't help $[\ldots]$ in order for them to move on to other sorts of treatment like enhancing their ability to cope on their own. [Dr regarding P2]

Further, there was a concern that introducing pain management ideas early could undermine the relationship, so doctors postponed discussion of pain management concepts, sometimes until medical interventions had been demonstrated to fail.

All the dyads that were mismatched on the most likely long-term treatment plan (subtheme 2.2: Table 1) were also mismatched on the likelihood that the next medical intervention would be effective for them (subtheme 2.1: Table 1). While they all agreed in the consultation about the next planned treatment (subtheme 1.1: Table 1) and most agreed on the doctor's description of extent of treatment effectiveness (subtheme 1.2: Table 1), patients' expectations of benefit exceeded the estimates given in the consultation and doctors' expectations were more skeptical about treatment effectiveness than conveyed in the consultation. For doctors, the possibility of a previously unidentified cause of the pain and an effective treatment for it persisted, even if unlikely:

I wouldn't be at all surprised if we get her spine X-ray back and it's not that bad [...] But I might be wrong! If however the scan shows a whopping great big disc that is pressing on her nerve, then I might say, "Oh well, let's try an injection, let's try surgery, as it might do". But if the scan shows nothing or virtually no disturbance, I would try and give her much more reassurance, and say the scan has got very minimal changes. [Dr regarding P4]

Doctors and patients, in different ways, seemed to focus on the hope that medical intervention would succeed, even where at the same time they acknowledged this as very unlikely. The understanding that the failure of these interventions, however predictable, clarified the future course of treatment was also shared by doctors and patients, but more in reflection during the interviews than explicitly in the consultation. Pain management remained, in many cases, contingent on disillusionment with medical techniques.

\section{Mismatch of patients' role within their treatment plan}

In all seven consultations where the role of the patient in treatment (subtheme 1.4: Table 1) was discussed, pain management was the only or next agreed intervention. This produced mismatches:
He came up and said, we can either offer you injection or acupuncture [...] It seems to me as though I am here [hospital] for ever more! [P6]

I actually think in the longer term, the most valuable thing we could offer her is the back pain management group, to actually sort of look at her expectations and goals, and try to make them realistic. [Dr regarding P6]

Five partial matches arose from patients understanding the broad imperative to keep mobile and to exercise, to which these patients had not agreed although their doctors thought that they had. The other partially matched dyads showed patients not recalling doctors' reported encouragement to increase their activity:

So she [doctor] has more or less told me to carry on with exercises that I am doing. [P8]

I think she can do more; I think she is a bit conservative, but hopefully I reassured her on that. [Dr regarding P8]

Another patient (P1) intended to rest in bed after the prescribed medical intervention, in the absence of other advice from the doctor. It seemed that both rationale and methods of pain management needed to be understood by patients for them to be likely to adhere to the treatment, given that patients' expectations of benefit from pain management were generally substantially lower than doctors' expectations (subtheme 2.1: Table 1).

\section{Mismatch of perceptions about patients}

Mismatches in this area (related to theme 3 ) occurred in only a minority of dyads, but since they emerged through doctors' spontaneous comments, it is possible that they might be true of more dyads than reported. Three clinically significant areas of mismatch are described.

First, in four instances, doctors overestimated how much the patient recalled of the consultation messages (subtheme 3.1: Table 1) and appeared to make unwarranted assumptions about what the patient already knew.

I think he understood that's where we are going [pain management], I'd be surprised if he was not clear on that. He's intelligent, works in IT or something. [Dr regarding P20]

Most patients reported that doctors asked them if they had any questions, but, in general, doctors reported not checking patients' understanding of the consultation messages but assuming that patients had understood.

Second, in three dyads, doctors spontaneously described the patient coping well with pain, inconsistent with the 
patient's self-report. Patients appear to have disclosed more about emotional difficulties and struggling with pain in the research interview than in the medical consultation:

I do struggle but I do carry on [...] I am alone, I'm scared, particularly when things go wrong, it's very very difficult to cope. [P7]

I think she is a very energetic dynamic lady who's very single minded and won't allow pain to stop her from doing things that she felt a commitment and responsibility to do.

[Dr regarding P7]

Third, in seven dyads, there seemed to be a partial mismatch between doctors' perceptions and patients' accounts of their hopes for the consultation. In two cases, doctors reported that the patient wanted a specific medical intervention, while patients described wanting whatever would best help them to function. One patient described how she had finally capitulated to trying an injection on the advice of a friend, which scared her, so raised it early in the consultation; the doctor heard this as a focus only on medical interventions and a lack of interest in pain management. Three further patients, described by doctors as having "fixed" ideas about treatment, had expressed preferences but had agreed eventually to try treatment that they had originally rejected.

\section{Discussion}

With relatively little research informing this field, there are some important positive points to note. FA, using patientidentified issues of importance, provided a novel and useful perspective on the content and outcome of the consultation. Overall, there appears to be reasonable concordance between doctors and patients, and patients were generally satisfied with their first consultation with a specialist. Satisfaction in consultations is consistently associated with shared decisionmaking, particularly over treatment, ${ }^{43}$ and among the topics discussed by all dyads, the highest level of agreement (all matches) was on the next planned intervention for pain following the consultation.

Three other areas showed no mismatch and more match than partial match: expectations of the next planned intervention; doctors' impressions of patients' understanding; and doctors' explanations of pain. Partial matches appeared to be more about completeness of detail rather than any systematic bias.

However, two topics discussed by all dyads showed substantial mismatch with no partial matches: the estimated likely (rather than expected) outcome of the next planned intervention and the long-term treatment plan assuming less than complete success of the next intervention. There was a distinct tendency for patients to be more optimistic than their doctors about success of the next treatment, even where they acknowledged wishful thinking, and to expect to receive continued medical interventions in the face of repeated failures. On the other hand, doctors planned to use the failure of medical interventions to justify a move to nonmedical rehabilitative physical and psychological interventions under the broad term of pain management. These longer term plans were the context for immediate treatment plans, so while there was agreement on what the immediate treatment was, there may not have been shared understanding about the longer term plans.

Among the topics discussed by nine or fewer dyads, two showed a mix of match, partial match, and mismatch: doctors' expectations of patient adherence and the patients' role in treatment; again, differences showed no systematic bias but differences in detail. However, two topics showed total mismatch: doctors' descriptions of patients' pain and doctors' descriptions of patients' agendas for the consultation. Doctors all underestimated patients' pain or overestimated their ability to manage it. On the patients' agenda, doctors underestimated patients' readiness to change ideas during the consultation and to be interested in nonmedical interventions, information, and rehabilitation.

The details of the consultation process appear to show that, although pragmatic aspects of treatment are fairly straightforward to convey (such as the distinction between medical interventions and a rehabilitative self-management approach), more complex issues often generate divergence of understanding or agreement. Two such themes which doctors and patients would probably agree are key to a fruitful consultation are the explanation of the nature of their chronic pain and what the patients have to do to manage it. Here, the consultation resulted more often in partial than full understanding and agreement, suggesting that more work is required to improve this process in the specialist consultation. While good communication - incorporating the patient's views and preferences into the consideration of possible treatments - contributes to satisfaction with the consultation itself, it is also associated with adherence to treatment, treatment completion, and better treatment outcome, ${ }^{44,45}$ all important in the complex journey of many people with chronic pain through treatment.

However, a further aspect of good communication ${ }^{46}$ is discussion of possible barriers to adherence, and here, we identified what might be the beginnings of non-adherence in some patients, albeit mostly non-intentional, when the doctor's message about activity was not heard or its implications 
understood. Main et $\mathrm{al}^{44}$ summarized the commonest shortcomings in medical consultations as failure to elicit patients' beliefs and expectations; failure to check patient understanding; and blocking of emotional disclosure. While we found little evidence of the first, there were certainly identifiable instances of doctors assuming rather than checking patient understanding, leading to partial matches and mismatches, in particular about long-term treatment plans.

It is harder to identify whether there was any blocking of emotional disclosure: patients described more difficulties in managing pain to the researcher than to their doctors, leading to doctors' overoptimism about patients' pain severity, impact, or manageability. This may be optimistic bias on the part of doctors but could have been at least in part an effect of the interview, since it followed the consultation in which patients had already partly articulated their difficulties. Additionally, the researcher being a psychologist rather than a doctor may have cued different material.

Perhaps, the issue of most concern, and one that troubles pain clinicians on a daily basis, is that doctors and patients find it difficult to agree. Doctors believe that the best option for patients is to accept that complete relief of pain is rare and that they are the main agents in their own rehabilitation. On the other hand, patients seemed not to anticipate this, nor to hear it when it was mentioned, but doctors also postponed a full explanation until medical interventions had failed. However, while doctors often believed that this point of failure was imminent - that it would follow the next agreed intervention - patients seemed to have less sense of either the radical change in focus or its imminence, unless they had already started on this course themselves. The problem may lie in expectations generated during the process of referral to the pain management team of effective resolution of their pain.

\section{Strengths and limitations of the study}

The only consultation investigated was the first meeting of the patient with the doctor. While some patients are discharged to primary care or referred on after a single consultation, many have one or more encounters with the same doctor or another in the same clinic, and understanding and concordance may develop over time and resolve the differences identified here. Further, we did not record the consultation but focused instead on the accounts shortly afterward provided by patient and doctor. We were interested more in how each party understood the content and process of the interaction rather than in establishing what was and was not said; nevertheless, a recording of each consultation would have provided valuable material for further analysis with which to understand partial matches and mismatches. While repeated interviewing of doctors about multiple consultations may have changed their behavior, we are unable to comment on whether this affected our results. We also did not analyze for gender, ethnic, or class match of patients to doctors: all can affect patients' beliefs and presentation. ${ }^{14}$

It is possible that some of our findings are particular to our clinic, its procedures and its culture. However, it was not difficult to relate our findings to existing concerns articulated in the literature, ${ }^{2,4-7,44}$ as well as in clinical discussions in the pain field, so we believe that the issues we raise are more general than local. Only replications in other specialist settings, and with larger numbers, will confirm or refute this. Although we have referred to communication skills, we chose not to analyze with reference to these. Useful as the framework may be for teaching future doctors, it is clear both that patients' and doctors' assessments differ quite considerably ${ }^{47}$ and that the contribution of communication skills to predicting outcome can be quite modest. ${ }^{48}$

\section{Clinical and research implications}

Despite the widespread recommendations to medical practitioners to check patients' understanding directly, ${ }^{14,31}$ also repeated with reference to communication about pain, ${ }^{44}$ it does not appear to be routine practice. Various considerations affect discussion of the longer term course of pain management, whether by medical means or by self-management. First, the doctor cannot be certain that the planned medical treatment will not resolve pain and does not want to undermine potential placebo effects by implying certain failure. Second, given this uncertainty and the patient's hopes of becoming pain-free, provisional discussion of what to do in the event of treatment failure seems to lack conviction on either side. The doctor is pessimistic about pain relief on the basis of odds; the patient is optimistic about pain relief on the basis of hopes and even superstition (the "magic" invoked by several patients). These divergent paths lead to further differences about the necessity, in the first appointment, of considering self-management methods and obtaining support in developing and refining them with the help of nonmedical team members. It may be that written or electronic information about possible pathways through interventions for the pain patient might help this discussion at the first consultation. We hope that our themes and subthemes may provide a focus for more detailed examination of shared and divergent experiences of consultations and their influence on the subsequent course of intervention. 


\section{Conclusion}

We were encouraged by the relatively high levels of satisfaction and trust among patients, their sense of having improved their understanding of their particular pain problem during the consultation, and their evident capacity to change their views and preferences as a result of information and discussion during the consultation. While medical information is now far more freely available through the World Wide Web than formerly, explanation and advice that are specific to that patient's history, pain, and circumstances remain highly valued.

Patients can learn or make fundamental changes in their understanding of the nature of chronic pain as a result of consulting a specialist. However, there remain mismatches of understanding during that consultation which could be amenable to rectification, either by checking the patient's understanding or by ensuring coverage of areas such as long-term outcomes. Teaching consultation strategy to pain specialists and educating patients on what to expect during an appointment are two ways that might lead to a more fruitful experience. Additionally, this research suggests that the pain specialists are not wholly aware when there are divergences in patient understanding. Sharing this and encouraging more reflective practice among doctors would change many specialists' current practice and bring about better outcomes in the management of long-term pain.

\section{Disclosure}

The authors report no conflicts of interest in this work.

\section{References}

1. International Association for the Study of Pain. IASP Taxonomy. Updated May 22, 2012. Available from: http://www.iasp-pain.org/ Taxonomy\#Pain. Accessed October 12, 2016.

2. Blyth FM, Macfarlane GJ, Nicholas MK. The contribution of psychosocial factors in the development of chronic pain: the key to better outcomes for patients? Pain. 2007;127:8-11.

3. Sprangers MA, de Regt EB, Andries F, et al. Which chronic conditions are associated with better or poorer quality of life? J Clin Epidemiol. 2000;53(9):895-907.

4. O'Brien EM, Roland M, Staud RM, et al. Patient-centered perspective on treatment outcomes in chronic pain. Pain Med. 2010;11(1):6-15.

5. Donaldson L. 150 Years of the Annual Report of the Chief Medical Officer: on the State of Public Health 2008. London: Department of Health; 2009

6. Eccleston C, Williams AC, Rogers WS. Patients' and professionals' understandings of the causes of chronic pain: blame, responsibility and identity protection. Soc Sci Med. 1997;45(5):699-709.

7. Geisser ME, Roth RS. Knowledge of and agreement with chronic pain diagnosis: relation to affective distress, pain beliefs and coping, pain intensity, and disability. J Occup Rehabil. 1998;8(1):73-88.

8. Newton-John TR. Psychological effects of chronic pain and their assessment in adults. In: Jensen T, Wilson PR, Rice ASC, editors. Clinical Pain Management: Chronic Pain. Vol. 2. London: Hodder Arnold 2002:101-111.

9. Leventhal H, Leventhal EA, Breland JY. Cognitive science speaks to the "common sense" of chronic illness management. Ann Behav Med. 2011;41:152-163.
10. Eccleston C, Morley S, Williams A. Psychological approaches to chronic pain management: evidence and challenges. Br JAnaesth. 2013; 111(1):59-63.

11. Sharpe M, Williams AC. Treating patients with somatoform pain disorder and hypochondriasis. In: Turk DC, Gatchel R, editors. Psychological Approaches to Pain Management: A Practitioner's Handbook. 2nd ed. New York, NY: Guilford Press; 2002:515-533.

12. Eccleston C, Crombez G, Aldrich S, Stannard C. Worry and chronic pain patients: a description and analysis of individual differences. Eur J Pain. 2001;5(3):309-318.

13. Ring A, Dowrick CF, Humphris GM, Davies J, Salmon P. The somatising effect of clinical consultation: what patients and doctors say and do not say when patients present medically unexplained physical symptoms. Soc Sci Med. 2005;61(7):1505-1515.

14. Weinman J. Healthcare professional-patient communication. In: Ayers S, Baum A, McManus C, editors. Cambridge Handbook of Psychology, Health and Medicine. Cambridge, UK: Cambridge University Press; 2007:435-439.

15. Woolf CJ. Central sensitization: implications for the diagnosis and treatment of pain. Pain. 2011;152(3 Suppl):S2-S15.

16. Williams AC, Eccleston C, Morley S. Psychological therapies for the management of chronic pain (excluding headache) in adults. Cochrane Database Syst Rev. 2012;11:CD007407.

17. Kamper SJ, Apeldoorn AT, Chiarotto A, et al. Multidisciplinary biopsychosocial rehabilitation for chronic low back pain. Cochrane Database Syst Rev. 2014;9:CD000963.

18. Crombez G, Van Ryckeghem DM, Eccleston C, Van Damme S. Attentional bias to pain-related information: a meta-analysis. Pain. 2013;154(4) 497-510.

19. Harris S, Morley S, Barton SB. Role loss and emotional adjustment in chronic pain. Pain. 2003;105(1-2):363-370.

20. Keefe FJ, Porter L, Somers T, Shelby R, Wren AV. Psychosocial interventions for managing pain in older adults: outcomes and clinical implications. Br J Anaesth. 2013;111(1):89-94.

21. Chou R, Fu R, Carrino JA, Deyo RA. Imaging strategies for low-back pain: systematic review and meta-analysis. Lancet. 2009;373(9662):463-472.

22. van Ravesteijn H, van Dijk I, Darmon D, et al. The reassuring value of diagnostic tests: a systematic review. Patient Educ Couns. 2012; 86(1):3-8

23. Froud R, Patterson S, Eldridge S, et al. A systematic review and meta-synthesis of the impact of low back pain on people's lives. $B M C$ Musculoskelet Disorders. 2014;15:50.

24. Loeser JD. Comprehensive pain programs versus other treatments for chronic pain. J Pain. 2006;7(11):800-801. [discussion 804-806].

25. Bonathan CJ, Zakrzewska JM, Love J, Williams AC. Beliefs and distress about orofacial pain: patient journey through a specialist pain consultation. J Oral Facial Pain Headache. 2014;28(3):223-232.

26. Darlow B, Dowell A, Baxter GD, Mathieson F, Perry M, Dean S. The enduring impact of what clinicians say to people with low back pain. Ann Fam Med. 2013;11(6):527-534.

27. Moseley GL, Nicholas MK, Hodges PW. A randomized controlled trial of intensive neurophysiology education in chronic low back pain. Clin J Pain. 2004;20(5):324-330.

28. Price C, Hoggart B, Olukoga O, Williams AC, Bottle A. National Pain Audit 2012. London: Dr Foster Research Ltd; 2012.

29. Peters S, Rogers A, Salmon P, et al. What do patients choose to tell their doctors? Qualitative analysis of potential barriers to reattributing medically unexplained symptoms. J Gen Intern Med. 2009;24(4): 443-449.

30. Petrie KJ, Frampton T, Large RG, Moss-Morris R, Johnson M, Meechan G. What do patients expect from their first visit to a pain clinic? Clin J Pain. 2005;21(4):297-301.

31. Khalib A, Farid A. Stopping doctor-patient communication gap: the ten essential methods. J Community Health. 2010;16(1):48-53.

32. Jellema P, van der Windt DA, van der Horst HE, Twisk JW, Stalman WA, Bouter LM. Should treatment of (sub)acute low back pain be aimed at psychosocial prognostic factors? Cluster randomised clinical trial in general practice. BMJ. 2005;331(7508):84. 
33. Kroon FP, van der Burg LR, Buchbinder R, Osborne RH, Johnston RV, Pitt V. Self-management education programmes for osteoarthritis. Cochrane Database Syst Rev. 2014;(1):CD008963.

34. McIntosh A, Shaw CF. Barriers to patient information provision in primary care: patients' and general practitioners' experiences and expectations of information for low back pain. Health Expect. 2003;6(1):19-29.

35. Ospina MB, Taenzer P, Rashiq S, et al. A systematic review of the effectiveness of knowledge translation interventions for chronic non-cancer pain management. Pain Res Manag. 2013;18(6):e129-e141.

36. Barker C, Pistrang N. Quality criteria under methodological pluralism: implications for conducting and evaluating research. Am J Community Psychol. 2005;35(3):201-212.

37. Ritchie J, Spencer L. Qualitative data analysis for applied policy research. In: Bryman A, Burgess RG, editors. Analyzing Qualitative Data. London: Psychology Press; 1994:305-329.

38. Ritchie J, Spencer L, O'Connor W. Carrying out qualitative analysis. In: Ritchie J, Lewis J, editors. Qualitative Research Practice: A Guide for Social Science Students and Researchers. London: Sage Publications Ltd; 2003:219-262.

39. White K [webpage on the Internet]. How Do Patients and Doctors Make Sense of Chronic Back Pain in the First Specialist Consultation? University College London Doctorate in Clinical Psychology thesis; 2012. http://discovery.ucl.ac.uk/1368818/. Accessed October 22, 2016.

40. Patton MQ. Qualitative Evaluation and Research Methods. Thousand Oaks, CA: Sage Publications; 1990.
41. Braun V, Clarke V. Using thematic analysis in psychology. Qual Res Psychol. 2006;3(2):77-101.

42. Joffe H. Thematic analysis. In: Harper D, Thompson AR, editors. Qualitative Research Methods in Mental Health and Psychotherapy: A Guide for Students and Practitioners. London: Wiley; 2011:209-223.

43. Lindhiem O, Bennett CB, Trentacosta CJ, McLear C. Client preferences affect treatment satisfaction, completion, and clinical outcome: a metaanalysis. Clin Psychol Rev. 2014;34(6):506-517.

44. Main CJ, Buchbinder R, Porcheret M, Foster N. Addressing patient beliefs and expectations in the consultation. Best Pract Res Clin Rheumatol. 2010;24(2):219-225.

45. Butow P, Sharpe L. The impact of communication on adherence in pain management. Pain. 2013;154(suppl 1):S101-S107.

46. NICE National Institute for Health and Clinical Excellence. Medicines adherence: involving patients in decisions about prescribed medicines and supporting adherence. Clinical guideline [CG76]. Published January 2009. Available from: https://www.nice.org.uk/guidance/cg76. Accessed November 8, 2016.

47. Kenny DA, Veldhuijzen W, van der Weijden T, et al. Interpersonal perception in the context of doctor-patient relationships: a dyadic analysis of doctor-patient communication. Soc Sci Med. 2010;70(5):763-768.

48. Pawlikowska T, Zhang W, Griffiths F, van Dalen J, van der Vleuten C. Verbal and non-verbal behaviour of doctors and patients in primary care consultations - how this relates to patient enablement. Patient Educ Couns. 2012;86(1):70-76. 


\section{Supplementary material \\ Interview schedule for patient interviews}

How did the consultation go?

What were the consultation's key messages?

What were the main messages the doctor said to you?

What did the doctor say about your pain?

- What did the doctor say about your pain now? How it might be in the future?

- Did this explanation make sense to you? Did it fit with how things are for you?

- What was the most important thing for you that the doctor said?

- Did the doctor tell you anything new or anything you had not heard before?

- Anything that surprised you or stood out for you?

- Anything that doesn't fit with your understanding of your pain?

- Did they say anything that confirmed what you already thought?

When you leave here who are you most likely to talk to or call up to tell about the consultation with the doctor?

- What do you think you will tell them about what was said in the consultation?

- How do you imagine they will react? What do you expect they will ask you?

- Is there anything you might not tell them about the consultation?

Was there anything you were not sure of or confused by in the consultation?

- Were there any questions you had that were not answered?

Has anything changed as a result of the consultation?

What did the doctor say was the next step for you?

- Did the doctor give you an idea of how effective this (treatment) would be?

- Did they mention any other treatment options?

- If says a medical intervention or another appointment - did the doctor give you an idea of anything that can help your pain until then?

- How was the decision made for you to (start a particular treatment/intervention)?

Has the consultation made any impact on:

- How you think about your pain?

- Anything you might do or not do?

- How you think about the future?

- Any decisions ahead of you?

How do you feel following the consultation?

What were you hoping for from this consultation?

- To what extent has this consultation met your expectations?

How has the consultation left you feeling?

- How are you feeling about (advice/messages given/treatment plan)?

- I was wondering what is most the upsetting/frustrating part for you ...?

- How are you left feeling about your pain?

Conclusion

Is there anything I haven't asked that you think might be important?

Do you have questions for me?

How have you found talking to me today? 


\section{Interview schedule for doctor interviews}

- From your perspective, what were the messages you said to the patient?

- What were the main messages that you wanted him or her to take away?

- How did you explain their pain?

- Were there any parts of the explanation that you think were more important for him or her to understand?

- Did you suggest anything that might help their pain?

- If yes - how did you describe the likelihood this (treatment/strategy) would alleviate their pain?

- Did you offer any other ideas?

- Do you think there is anything else he or she could do to help his or her pain? If yes - did you discuss this with him or her?

- How much do you think he or she understood or took on board what you said?

- What impact, if any, do you think the consultation will have on how he or she thinks or feels about their pain?

- What impact, if any, do you think it will have on anything that he or she plans to do?

- If advice given - to what extent do you think he or she will follow your advice?

- How do you imagine he or she left the consultation feeling?

- Looking back, are there any parts of the consultation you would have done differently?

- Is there anything that I have not asked, that you think might be important about the consultation?

\section{Publish your work in this journal}

The Journal of Pain Research is an international, peer reviewed, open access, online journal that welcomes laboratory and clinical findings in the fields of pain research and the prevention and management of pain. Original research, reviews, symposium reports, hypothesis formation and commentaries are all considered for publication.
Dovepress

The manuscript management system is completely online and includes a very quick and fair peer-review system, which is all easy to use. Visit http://www.dovepress.com/testimonials.php to read real quotes from published authors. 